\title{
Grupo de Mujeres en Situación de Maltrato. Evolución metodológica
}

\section{Group of Abused Women by Their Partners. Methodologic Development}

\author{
Júlia Masip, Concha Pont y Alicia Salaberry
}

Ayuntamiento de Barcelona

\begin{abstract}
Resumen. El presente artículo pretende resumir diez años de experiencia y enriquecimiento del extenso equipo de profesionales que interviene en el trabajo práctico de tratamiento del grupo de mujeres en situación de maltrato, iniciado a través de la atención directa y dirigido a las mujeres que sufren maltrato y que forman parte de un territorio concreto y común. El artículo, fruto de la experiencia diaria y directa, aporta aspectos importantes en el proceso de evolución de la experiencia desde hace 10 años, el enriquecimiento por parte del equipo de profesionales y, sobre todo, la mejora de la autoestima y autonomía en la valoración de las mujeres integrantes. Se cumplen los objetivos de la intervención grupal y comunitaria, innovando en la fase final del cierre con la participación en la conducción de las propias mujeres.

Palabras clave: intervención grupal, trabajo en equipo, mujeres en situación de maltrato, proceso temporal, fortalecimiento, autoestima y autonomía.
\end{abstract}

\begin{abstract}
This experience article pretends to visualize - in a graphic and active mannerthe work of an extended team of intervention group. It is addressed to abused women at the hands of their partners and belonging to as specific and common territory; contributing with important aspects to the evolution process of the experience from ten years, as well as the enrichment of the professional team and specially the improvement on the self esteem and autonomy in the valuation of the group women, accomplishing the aims of the communitarian and intervention group with the introduction in the final phase of women participation. Key words: intervention group, work team, abused women, temporary process, strengthening self esteem and autonomy.
\end{abstract}

\section{Introducción}

La intervención grupal, en la que se centrará este artículo, sigue el proceso, la evolución y el crecimiento tanto de la mejora del grupo en sí y sus resultados, como del crecimiento profesional de las conductoras y observadoras. Presentamos esta intervención grupal como una experiencia práctica que funciona, sus mejoras, su evolución y también la impor-

La correspondencia sobre este artículo debe enviarse a la primera autora al Centro de Servicios Sociales de Guinardó. Ronda Guinardó, 113-141.08041 Barcelona. E-mail: jmasip@bcn.cat tancia de formar parte de un proyecto global que comporta tanto para las mujeres como para las profesionales que lo forman un trabajo en red, una proyección comunitaria y de ciudad (Roca y Masip, pendiente de publicación, 2010)

El proyecto global parte de las demandas en atención directa de mujeres residentes en el Distrito de Horta-Guinardó de la ciudad de Barcelona ${ }^{1}$ que sufren maltrato por parte de sus parejas. Desde

\footnotetext{
${ }^{1}$ Población total del Distrito: 170.135 personas, de las cuales 89.800 son mujeres. SIBSS (Sistema Informático Básico de Servicios Sociales. Datos del Ayto. de Barcelona, 2007-2008).
} 
1999, la metodología grupal se aplica de manera rotativa entre las diferentes profesionales de las cuatro zonas del Distrito: Carmelo, Horta, Guinardó y Vall d'Hebron. El grupo lo conducimos una trabajadora social y una psicóloga, más otra asistente social ejerciendo de observadora aprendiendo desde la práctica, nos acompaña como transcriptora una alumna en prácticas de psicología de la Universidad de Barcelona.

\section{(SIBSS. Sistema informático básico de Servicios} Sociales. Datos del Ayuntamiento de Barcelona, 2007-2008)

El grupo de mujeres se complementa con un espacio infantil para los hijos-as de las integrantes, conducido por una monitora y una trabajadora familiar. Dicho grupo forma parte de la intervención comunitaria que complementa la proyección integral hacia toda la población femenina. Dicha intervención comunitaria fue coordinada inicialmente por dos psicólogas sociales y comunitarias -una de ellas coautora de este articulo-, que, a partir de las mujeres afectadas del primer grupo de intervención, también con la participación de las asociaciones y el Consell de la Dona del Distrito, y de los-as profesionales de la red, creó un material de detección, sensibilización preventiva y difusión -un cuaderno para profesionales y un cuento para las mujeres, Cenicienta tiene un mal sueño, J. Masip, Ch Martinez (2002) -, que todavía funciona y se utiliza como material de trabajo en la dinámica grupal.

\section{Objetivos generales de la intervención}

\section{Configuración del equipo}

En cada zona del Distrito hay un equipo de base, formado por la directora del Centro de Servicios Sociales, trabajadoras-es sociales, educadores-as sociales, uno-una psicóloga, una recepcionista, una administrativa y trabajadoras-es familiares (estas últimas a través de una empresa externa). Se considera importante que la conducción del grupo se realice por dos profesionales, la psicóloga, como experta en aspectos psicológicos de sufrimiento emocional y en dinámica grupal, y la trabajadora social, como experta en gestión de recursos para mujeres y también en dinámica grupal. Se da la máxima importancia a sumar y compartir la intensidad de la problemática, al mismo tiempo que la observadora garantiza la formación grupal-vivencial continuada y la rotación como conductora en el próximo grupo.

\section{Creación del grupo a nivel de Distrito}

La propuesta del Distrito es crear y configurar un grupo al año, de manera rotativa en las diferentes zonas. La experiencia que exponemos, se crea y ubica en la zona del Guinardó , siendo el sexto grupo desde que se creó el primero, en el curso 1999-2000.

\section{Afianzar y mejorar el trabajo de circuito y de red}

Se actualizan el documento de presentación del nuevo grupo y la ficha de derivación, al mismo tiempo que se difunden estos documentos a todos los servicios del Distrito (ambulatorios, centros de salud mental, centros psicopedagógicos, centro de atención a la mujer, centros de formación y entidades privadas que trabajan con mujeres) y a los cuatro centros de servicios sociales. De esta manera se pretende afianzar y mejorar el trabajo en red, llegar al máximo de mujeres posibles, ateniendo muy de cerca las derivaciones y los servicios desde dónde llegan, siendo ésta una parte muy importante e imprescindible para la creación del nuevo grupo. Al mismo tiempo, se trabaja para la unificación de criterios entre todos los profesionales que forman la red, buscando objetivos y resultados comunes.

\section{Reforzar la autoestima y disminuir la situación de maltrato de las mujeres integrantes del grupo}

La autoestima es el eje transversal de toda la intervención. Es un valor esencial que se fomenta desde el principio al final de todas las sesiones y en todos los ámbitos: la infancia, la familia de origen, 
las relaciones afectivas, la formación recibida, el trabajo, la salud, las amistades, el ocio, etc. En primer lugar, es importante que la mujer sea consciente de que está en situación de sufrir o haber sufrido maltrato por parte de su pareja o ex - pareja y que todavía sufre secuelas que no le permiten avanzar.

A partir de ahí, se concreta el contenido de la intervención: la preparación de las sesiones, ejercicios, aportaciones de otros profesionales, la escucha de los relatos, el análisis de las causas, la búsqueda de alternativas y refuerzos para que las mujeres superen los maltratos a través de sus propias experiencias, búsquedas de soluciones y reflexiones también a través de las aportaciones de las profesionales que conducen el grupo, les cuales llevan unos cuantos años de experiencia en la evolución de esta problemática. El objetivo que se persigue y pretende después de 20 sesiones es disminuir el maltrato y mejorar la situación de las mujeres. Este es el objetivo más importante a conseguir dentro de la intervención grupal.

\section{Desarrollar la independencia y la autonomía de las mujeres}

De la misma manera que durante todo el proceso grupal se trabajan el empoderamiento/fortalecimiento y la autoestima, también forman parte importante de la intervención la independencia y la autonomía. Pero es sobre todo en la última fase, hacia la quinceava sesión, cuando la incorporación de las mujeres en la conducción grupal se introduce dentro del cierre grupal y trabajamos el proceso de duelo (ver metodología).

\section{Metodología}

En octubre de 2006, las profesionales configuramos el equipo iniciando las reuniones de trabajo (dos horas semanales). El primer paso es actualizar el documento de presentación del nuevo grupo que acompaña las fichas de derivación de mujeres para el grupo a otros servicios; guión de la primera entrevista para la inclusión en el grupo y todo el resto de material; fichas de seguimiento para las sesiones y nueva revisión de las situaciones de no-inclusión.
A partir de las fichas enviadas por los/las profesionales de los servicios derivantes, el equipo va citando telefónicamente a las mujeres derivadas que son susceptibles de integrarse en el grupo.

Programamos entrevistas compartidas por la trabajadora social y la psicóloga en función de la complicación y/o confusión que nos presente la ficha recibida. El equipo valora conjuntamente la idoneidad y aceptación de las mujeres susceptibles de formar el grupo, siendo el número máximo de diez mujeres.

\section{Circuito de derivaciones}

Una vez revisado el material a utilizar se hace llegar la ficha de derivación a través del correo a los cuatro centros de Servicios de Atención Primaria y a los diferentes servicios antes mencionados. Los profesionales referentes rellenan la ficha y la retornan a través de e-mail, correo interno, fax, o en mano si son compañeras del propio Centro. En este grupo se recibieron 28 fichas. Al principio costó mucho: a los profesionales de los diferentes servicios les costaba enviar las fichas y no siempre las situaciones eran adecuadas (el maltratador era el hijo, o estaba en el hospital, o la mujer no tenia clara su participación en el grupo, o no coincidía en el horario, o encontró trabajo...).

Tuvimos que realizar mucho trabajo telefónico y de coordinación con los-las profesionales referentes para poder convocar a las mujeres a la primera entrevista de inclusión: 12 entraron en el grupo y 16 fueron descartadas.

\section{Entrevistas para la idoneidad al grupo}

Como anteriormente hemos expuesto lo idóneo en la primera entrevista es que esta sea realizada por las dos profesionales conductoras, pero en este grupo tuvimos que repartir las entrevistas entre las tres profesionales, incluyendo también la observadora por la cantidad de derivaciones (28) recibidas. En algún caso excepcional, por la complejidad de la situación, se hizo necesaria una segunda entrevista con dos profesionales. 
Los objetivos de esta primera entrevista son contrastar la información recibida, ampliarla, actualizarla, conocer la opinión de la mujer ante la posibilidad de integrarse en un grupo, informarla de la posibilidad del espacio infantil y saber si va hacer uso de él, y sobretodo valorar la idoneidad de las mujeres. Por ello descartamos, para el buen funcionamiento del grupo, que no sufran una patología mental severa, ni toxicomanía, ni deficiencia mental. Decidida la idoneidad, se le informa de la fecha aproximada de inicio del grupo, horario, día y lugar.

\section{Preparación y previsión de los ejercicios corporales}

Como resultado de la reflexión y valoración de las primeras experiencias grupales -las mujeres salían del espacio con mucha carga emocional y con dificultad para cerrar los temas, pidiendo más espacio, consultando frecuentemente fuera del espacio grupal a las profesionales y sintiéndose con dificultad para la despedida-, se incorporó el espacio corporal en la primera experiencia grupal específica de maltrato en el año 2001-2002.

Las profesionales observamos un alto porcentaje de mujeres con graves dolencias físicas, desde trastornos menstruales a diversos tumores de la biología femenina -mama y aparato reproductorademás de dolencias de espalda, cervicales, lumbares... y también respiratorias. A nivel preventivo, se planteó cerrar las sesiones con un espacio de ejercicios respiratorios y de movimiento ayudando a movilizar el riego sanguíneo que pudiera contraer la narrativa y escucha de relatos tan dolorosos emocionalmente. Se trata de ejercicios de qigong, su duración es de 20 minutos y están guiados por un médico especialista en medicina clásica china, el cual hace un primer diagnóstico y orienta durante todo el proceso a las mujeres sobre los ejercicios más convenientes para sus dolencias $\mathrm{y}$ dificultades físicas. Son ejercicios muy bien aceptados por las mujeres, ya que rebajan el alto nivel de contenido emocional, diluyen el dolor y cierran el discurso verbal, facilitando la despedida.

\section{Espacio Infantil}

Paralelamente a la construcción del grupo de mujeres, se crea el espacio infantil, preveyendo su configuración, cantidad de niños, edades, reserva de espacio, material necesario y sobre todo la coordinación intra-equipos.

En este grupo en concreto fueron muy pocos los niños-as integrantes del espacio infantil, y de muy corta edad: dos niñas de 3 años, un bebé de 5 meses y otra bebé de 20 meses. En alguna que otra sesión, una de las mujeres, la cual no finalizó el grupo trajo también alguno de sus hijos de corta edad (4 años) con algún amiguito, teniendo que recordar a las madres los objetivos del espacio infantil entendido como espacio específico de observación, contención y valoración respecto a la influencia de la situación materna hacia sus pequeño-as.

Se trabajó la dificultad de separación de las madres porque restaba tiempo a su participación en el grupo. Otro aspecto que se trabajó mucho, y que se trató con especial cuidado, fue la información respecto al grupo que la mujer daba a su pareja, padre de sus hijos-as, para presentar el espacio infantil.

Generalmente, sin consultar a las profesionales, las propias mujeres prefieren no hablarles a sus parejas/padres sobre el espacio infantil, dando diversas explicaciones (ludoteca, espacio de juego...) sin introducir el tema del maltrato, para prevenir cualquier situación de prohibición a los hijos, lo que significaría que la mujer tampoco podría acudir a las reuniones. Concretamente en este grupo ocurrió una situación en que la madre de dos niñas - una de 3 años y una de 20 meses- sí le explicó a su pareja la finalidad del grupo y del espacio infantil. Sin avisar, el padre vino a buscar a sus hijas, sin que ni la madre ni las profesionales del grupo de mujeres y del de los niños tuviesen conocimiento previo. Las monitoras avisaron a la madre, que no impidió que el padre se las llevara, y las niñas ya no volvieron más. Esta situación se expuso y se trabajó en la siguiente sesión. La madre explicó que no se sintió suficientemente fuerte para confrontar el hecho con el padre de las niñas y así poder recuperar el espacio infantil.

No es un espacio fácil, ni para las monitoras ni para las madres, aunque los niños-as hacen muchos 
cambios positivos: aprenden a mejorar relaciones sociales pasándoselo bien a través del juego.

Todas las profesionales de los dos espacios se coordinan para ir valorando la evolución y poder hacer una devolución de la monitora del espacio infantil a las respectivas madres, de manera individual en la sesión final del grupo.

\section{Material que utilizamos}

Los diferenciamos en dos tipos:

1) Material que utilizamos y creamos específicamente Inter-profesionales:

Cuento detector "Cenicienta tiene un mal sueño. Apuntes para despertar y salir del cuento." J. Masip, Ch. Marinez(2002). Hoja de presentación del grupo a otros Servicios de red. Ficha de derivación.

Intra-profesionales (equipo de conducción): Listado de derivaciones de casos para el grupo, zona, servicio, entrevista e idoneidad. Guión de entrevista individual. Cuadro de objetivos de cada sesión. Calendario de participación. Ficha de valoración de la sesión -por la observadora-.Ubicación de las mujeres en la sesión. Cuestionario de evaluación del grupo.

2) Material para el grupo, ya utilizado en anteriores grupos, buscado y/o creado específicamente para algún tema del grupo actual, también que puedan aportar las propias integrantes: Fotocopias de artículos de revistas, diarios. Material de Qi-Gong. Otros cuentos o relatos. Recortes, fotos para construir "collages". Retornos (escritos, cuadros) de sus ejercicios y aportaciones con las que podamos trabajar.

\section{Intervención}

La intervención no empieza cuando se inicia el grupo, sino mucho antes, de septiembre-octubre a diciembre, para revisar material y documentación, enviar y recoger las fichas de derivación y realizar

Cuadro con las diferentes características de las mujeres

Las variables que componen el cuadro en vertical, son: Inicial del nombre de las 9 mujeres. Zona del territorio ( Gu=Guinardó 66’6\%,Horta 22’2\% y Carmelo 11\%). Servicio que la derivó (S.S= Servicios Sociales 55\%, Salud 22\%,Centro privado11\% y Salud mental 11\%). Edad de la mujer, de 29 a 73 años. País de origen (Españolas 55\%, latinoamericas 44\%: Uruguai, Colombia, Ecuador y Bolivia). Convivencia (44\% si, 55\% no) con la pareja maltratador. Hijos en convivencia $10=52$ ' $6 \%$ o independizados $9=47 \%$. Trabajo(22\%si, 72\%no). Salud (55\% enfermas y $44 \%$ sanas) ,las tres mujeres más mayores tienen enfermedades bastante graves: problemas respiratorios, hipertensión, fibromialgia y depresión. Por último el tipo-s de maltrato (físico $78 \%$, psicológico 100\%, sexual 44\%, económico $89 \%$ y social $66 \%$ ).

\begin{tabular}{|c|c|c|c|c|c|c|c|c|c|}
\hline Mujer & $M$ & $I$ & $K$ & $R 1$ & $R O$ & $D$ & $R 2$ & $G$ & $E$ \\
\hline Zona & Guinardó & $\mathrm{Gu}$. & Horta & $\mathrm{Gu}$. & Carmelo & $\mathrm{Gu}$. & Horta & $\mathrm{Gu}$. & $\mathrm{Gu}$. \\
\hline S. derivante & Salud & salud & S.S. & Privado & $S . S$ & S.S. & $S . S$. & S.Mental & S.S. \\
\hline Edad & 73 & 61 & 56 & 43 & 40 & 32 & 32 & 32 & 29 \\
\hline País & Esp & Esp. & Esp. & Esp. & Urugu. & Colm. & Ecua. & Esp. & Boliv. \\
\hline Convivencia pareja & si & si & si & no & no & no & no & si & no \\
\hline Hijos & 2 & 5 & 2 & - & 2 & 1 & 4 & 2 & 1 \\
\hline Trabajo & no & si & si & no & no & si & no & no & no \\
\hline \multirow[t]{2}{*}{ Salud } & prob/ & Sordera & fibro. & Depre. & - & - & - & depre. & - \\
\hline & graves & hipert. & Depre. & & & & & & \\
\hline Tipo de & Psi. & Fis. & Fis. & Fis. & Fis. & Fis. & Fis. & Psi. & Fis. \\
\hline \multirow[t]{4}{*}{ maltratos } & econ. & Psi. & Psi. & Psi. & Psi. & Psi. & Psi. & Econ. & Psic. \\
\hline & sex. & Sex. & Econ. & Econ. & Econ & & econ. & Social & Econ. \\
\hline & social & econ. & Sex. & Social. & Sex & & & & Social \\
\hline & & social & & & social & & & & \\
\hline
\end{tabular}


las entrevistas oportunas. Y ya en enero se perfila el grupo y empezamos a preparar la primera sesión y el espacio infantil, siempre en función de las características de las mujeres que lo integrarán.

Al ser 20 las sesiones, muchas para explicar una por una, realizamos un análisis por etapas temporales. La primera comprende desde la primera hasta la $9^{\text {a }}$ sesión, cuando cerramos las nuevas incorporaciones. Analizamos la creación del grupo, cohesión, relatos iniciales, dolor y sufrimiento compartido, contención. Es la etapa más difícil, por el contenido inicial de los relatos, por la ansiedad hacia el grupo, por las expectativas creadas y el enfoque de las profesionales.

Una segunda etapa comprende de la $10^{\mathrm{a}}$ a la $16^{\mathrm{a}}$ sesión, que se inicia con el retorno de las vacaciones de Semana Santa, cuando se ha dado un tiempo de separación, también de reflexión, cuando las mujeres ya empiezan a ser más conscientes de los efectos grupales. Tienen ganas de reencontrarse, siendo esta etapa más productiva, de reparación y de contacto con su propio yo, dejando atrás la victimización y la rabia hacia el otro (el maltrato de su pareja). El grupo adquiere cuerpo e identidad, surge el nombre del grupo, las propias mujeres lo llaman "Lo mejorcito para él", también "Desecha la sombra que te oprime y busca la luz". En esta etapa se dan fugas.

La tercera y última etapa, desde la $17^{\mathrm{a}}$ a la $20^{\mathrm{a}}$ sesión, momento de cierre pero a la vez de obertura, ya que iniciamos la participación activa de las mujeres en la conducción y cierre del grupo, con el objetivo de consolidar la autonomía, dar mayor seguridad e independencia una vez finalizado el grupo.

\section{$1^{\text {a }}$ etapa: exposición, relatos y cohesión grupal "sumergirse en el grupo"}

La primera sesión se considera crucial para establecer el clima que se quiere crear, un clima de confianza, objetividad, confidencialidad, positivismo, etc. Por ello se prepara con mucho cuidado: se colocan las sillas previsibles a ocupar, en forma de herradura. Las profesionales se colocan juntas en la parte central y las mujeres alrededor. A medida que van llegando, las profesionales las acogen y antes de las presentaciones, se les hace rellenar un cuestionario de autoestima (Escala de Autoestima de Rosenberg/ 1965).

Las profesionales se presentan y explican el porqué del grupo; exponiendo su experiencia y el proyecto global de Distrito, las situaciones similares que traen las participantes al grupo y los elementos curativos de este* Pichon Rivière, (1985). Al mismo tiempo, se encuadran las normas, horarios de la sesión grupal , calendario, grupo abierto a nuevas incorporaciones hasta Semana Santa, compromiso y puntualidad, escucha, respeto, silencio -sin móviles-, confidencialidad, parte corporal y ejercicios físicos, posibles abandonos y explicación del espacio infantil.

Seguidamente se prepara su presentación; en este caso se intentó hacer una presentación por parejas, preguntándose mutuamente sus nombres, edad, situación familiar, hijos, trabajo, salud, el porqué de su participación y expectativas en el grupo. Les costaba compartir ya desde el inicio, algunas mujeres salían porque no podían contener la emoción que se genera, muy condensada, no podían parar de llorar, por lo que acabaron presentándose ellas mismas. Sus relatos eran impactantes, sobre todo el de las más mayores que acumulan tantos años de sufrimiento.

En este momento las profesionales tienen que hacer un gran esfuerzo para contener tanta emoción psicológica, aprovechando para explicar el sentimiento y deseo de abandonar el grupo que puede surgir a partir de la escucha intensa de tantas situaciones graves y similares a la vez (supuesto de ataque y fuga de Bion, 1980), y tranquilizándolas con la importancia de comprometerse a asumir la confidencialidad e incitándolas a continuar, explicando las dificultades iníciales y también el propósito de avanzar con ellas. Para distanciarse del duro relato inicial, se pone en práctica el ejercicio de grupo nominal para extraer los temas más importantes a trabajar. Por orden de mayoría de votos:

Autoestima, relación con los hijos, seguridad, perder el miedo y poder afrontar situaciones de crisis, salud, sentimientos, convivencia, economía, control, vinculo familiar, hábitos, verbalizar los maltratos, valores, límites, reemprender el futuro y reparar heridas, herencia y causas, compartir. 
Además de sus aportaciones, también el compartir y recibir, que se trabaja en función de su demanda. Las profesionales se convierten en meras transformadoras que elaboran sus aportaciones y demandas para que ellas avancen, utilizando la técnica de "grupo nominal" para concretar sus demandas y aportaciones: se quiere conseguir que el grupo adquiera una dinámica creativa, positiva y participativa.

Por último, se cuida al máximo el cierre de la sesión, preguntando una por una a todas las participantes cómo se han sentido y si han podido expresar lo que querían; se les pide sus teléfonos para saber de ellas en caso de que no vengan o quieran comunicarse algo. En general retornan que se han sentido a gusto y parecen más tranquilas que al principio, con una expresión facial más relajada. A continuación se inician los ejercicios físicos de Qi-gong, dándoles al final un dosier con la explicación de los ejercicios a trabajar.

La primera etapa es muy florida en cuanto a exposiciones y relatos: a través de ellos todas se van conociendo. Y también de muchos ejercicios: la margarita de la autoestima, collage de fotos históricas y actuales, etc. Es una etapa de entradas y salidas, donde poco a poco, se valora dentro del mismo grupo, porque algunas mujeres no vuelven y se incorporan nuevas entradas. Realizamos un trabajo de despedidas y acogidas, manteniendo a las mujeres más constantes que, a su vez, acogen a las que llegan llorando, explicándoles los beneficios grupales. En esta etapa se va creando la cohesión grupal y también van surgiendo alianzas, se empiezan a romper las dinámicas preparadas por las profesionales para introducir la propia, la de las mujeres, con sus vivencias y sus actuaciones... el grupo empieza a funcionar por si mismo.

\section{$2^{\mathrm{a}}$ Etapa, de la $10^{\mathrm{a}}$ a $16^{\mathrm{a}}$ sesión, reflexión post- vacional, reencuentro, conciencia de grupo, reparación y empoderamiento}

Esta segunda etapa se inicia invitando a una profesional externa al grupo, una abogada.

Ya en las primeras sesiones, las profesionales conductoras preguntamos a las mujeres qué temas especializados quieren consultar y se recurre a los profesionales externos adecuados. Con los años se ha ido consolidando la preocupación por los temas de salud y nueva legislación, por lo que se invita al médico que dirige el espacio corporal de qi-gong para que dedique una sesión a consultas físicas y salud femenina, haciendo especial referencia a los efectos causados por los maltratos recibidos.

Se reserva otro espacio para una abogada, con la que se contacta a través del PIAD (Punto de Información y Atención a la Mujer) con el que colaboran las abogadas gratuitamente. Fueron consultas legales y jurídicas, ya que algunas de las mujeres se encuentran dentro de un proceso judicial, sea separaciones, denuncias o simplemente quieren informarse de sus derechos y de la evolución de las leyes que las amparan.

Se estructura la sesión en cuatro bloques: presentación de la profesional, en esta ocasión miembro de Mujeres Juristas, especialista en violencia y legislación de genero y que asesora a mujeres desde el Centro de Atención a las Mujeres del Distrito. Presentación de las mujeres que concretan sus consultas, y devolución de la abogada a las consultas, más ampliación de explicaciones generales sobre violencia de género: orden de alejamiento, protección, separaciones, derechos y avances de las leyes para mujeres.

En esta etapa las mujeres se sienten más tranquilas, más seguras y escuchadas. Se procura que el clima grupal permita a las últimas mujeres incorporadas un "tempus" para que puedan alcanzar el ritmo en el que está el grupo y poco a poco puedan ponerse al mismo nivel que el resto de las participantes. El grupo está más abierto a compartir su experiencia fuera del espacio grupal, aceptando participar en un estudio de la Universidad de Barcelona sobre un cuestionario de maltratos psicológicos. Todas se suman a un principio de solidaridad que va más allá del grupo.

Es el momento en que las participantes traen el maltrato presente, diario, al grupo, desean compartirlo y piden consejo a sus compañeras y a las profesionales. El grupo, por si mismo, hace de contención en situaciones o relatos graves (Pichon Ribière, 1985).

Ejemplo de una situación crítica: Una de las 
mujeres tenia pensamientos de "acabar con todo, tomándome pastillas, cortándome las venas o irme de casa ". El grupo la contiene, le da otras opciones: “¿porqué no te vas a vivir con tu hija?”, “¿porqué no se va él?". La anima a denunciarlo.

Los temas tratados en esta etapa son: herencia familiar, sexualidad, perder el miedo y poder afrontar, salud, economía, control, vínculos afectivos, verbalizar los maltratos, valores y límites. Los ejercicios de rol-pleying, que acompañaron los temas, son los más impactantes y donde se sienten más sorprendidas, más reconocidas y donde empiezan a relacionar causa- efecto.

En esta etapa, el grupo ya se permite quedar fuera del espacio grupal formal para tomar un café antes del inicio de la sesión ,y se invitan por sus aniversarios.

También se producen dos fugas en esta etapa, que fueron tema de supervisión, una por grave situación económica y de vivienda, la otra por dificultades de afrontar entre otros temas su problema de bloqueo sexual (abusos y violación por parte de su expareja).

Al mismo tiempo esta etapa nos enlaza con las últimas sesiones, las de cierre, de futuro, de obertura y de conducción-participativa para reforzar su seguridad, autoestima y potenciarles con la conducción del grupo por ellas mismas, su independencia y autonomía.

\section{$3^{a}$ Etapa, de la $16^{a}$ hasta la $20^{a}$ y última sesión. Etapa de cierre, independencia y futuro}

Se les concreta como será su participación en el cierre, conduciran por parejas. Las profesional escogemos las parejas pensando en los tema más adecuados para trabajar cada una, según las dificultades que les hemos observado.

Son las profesionales las que organizan y preparan esta parte: cuando se lo proponen, las mujeres muestran fuertes resistencias:

-No, no sabremos hacerlo, no lo hemos hecho nunca.

No quieren que se acabe el grupo y se resisten a cambiar su posición pasiva, les cuesta creer en sus potenciales; aunque se les propongan temas fáciles (de futuro, de amistad, laboral) se muestran aún dudosas e incapaces.
Para no incrementar las resistencias de las mujeres a las sesiones conducidas por ellas, la siguiente sesión es conducida por las profesionales. Para tranquilizarlas se les informa de que la observadora se quedará con ellas durante la sesión para seguir con la trascripción y asegurar la evolución de la dinámica, pero no participará en ningún momento. Las profesionales entran al inicio de la sesión para enmarcar los objetivos y al final de ésta para evaluar cómo se ha desarrollado la sesión, la conducción y el tema, preguntando también las mujeres a sus compañeras conductoras como se han sentido y evaluándolas. Se aprovecha para reforzarlas en su nuevo rol de conducción, para motivar a la siguiente pareja en la preparación y que se sientan fuertes, para diluir las resistencias que presentan algunas cuando no preparan nada e improvisan en función de las necesidades del grupo y las suyas propias, siguiendo en una posición pasiva. Otras sí lo preparan, buscan documentación y participan más activamente de esta fase final.

A las profesionales, esta propuesta nos da mucha información, además de promover la participación para reforzar su autonomía, su seguridad y - esta es la parte más importante y por la que se propone esta metodología - su independencia del grupo y personal, puesto que en otra experiencias, les costaba mucho aceptar la fase final y la despedida, tanto de las profesionales como de las compañeras integrantes, y el duelo era muy intenso, triste y dependiente.

También se supervisó esta etapa, revisando el poder de la conducción, las habilidades, su posición, dándoles feed-back se hizo una sesión de ensayo, proporcionándoles pautas por parejas y así se iban preparando. Poco a poco se iban introduciendo, incorporando a la dinámica del cierre como una forma más positiva, sintiéndose capaces de conducir a sus propias compañeras sin presencia de las profesionales, que procuran no intervenir en su proceso de avance y evolución dentro de la sesión, como camino hacia su autonomía futura y a la vez reforzando su independencia, igual que se refuerza hacia sus parejas o ex-parejas.

Se hizo una fiesta final con retorno poético (un poema personalizado para cada mujer que recoge sus aspectos más positivos y a mejorar), regalos, merienda y fotos del grupo, cerramos dando por 
finalizada la intervención grupal. Cuando acaba el grupo se realiza un retorno a las profesionales de referencia con las que todas siguen en tratamiento.

A los 6 meses se realiza otra sesión para evaluar su situación.

\section{Resultados y evaluación}

Para poder extraer resultados a nivel cuantitativo les pasamos un cuestionario revisado de satisfacción y valoración, utilizado en otros grupos anteriores.

El $86 \%$ del total de las mujeres respondieron a todas las preguntas.

Las respuestas más significativas fueron:

- En cuanto a la valoración general del grupo: el $66 \%$ muy adecuado, el $33 \%$ restante, bastante adecuado.

- Los aspectos mejor valorados fueron: Los ejercicios y trabajos dentro del grupo: $66 \%$ muy adecuados. La profesional externa-abogada: $83 \%$ muy adecuada. Las aportaciones más personalizadas: $66 \%$ bastantes adecuadas.

- En cuanto a valoraciones negativas, sorprenden las resistencias a dirigir el grupo: el 50\%, lo consideraron "poco adecuado". Igual que también el $50 \%$ no cambiaría NADA. El mismo porcentaje $(50 \%)$ aumentaría el número de las sesiones.

- En cuanto al espacio, número de asistentes, el entorno y el horario, el $66 \%$ lo considera muy adecuado, siendo el número de asistentes al grupo, con un $83 \%$, el aspecto más valorado.

- Los aspectos valorados negativamente son la duración: el tiempo, que siempre les parece corto y poco (siendo 20 sesiones, en seis meses), y el espacio grupal infantil; el 16'6\% lo valoraron poco adecuado. Ya sabemos que en este grupo nos costó mucho encontrar las profesionales adecuadas: no había educadores-as especializados y la trabajadora familiar faltó en algunas sesiones, siendo siempre sustituida, pero es importante que las figuras sean fijas y de referencia para los niños-as, dando más confianza también a las madres.

En cuanto a temas trabajados, los más puntuados, por orden de mayor a menor, fueron:
- Saber reaccionar y miedos a sufrir daños: $83 \%$

- Ganas de vivir e ilusiones: 66\%

- Autoestima: $50 \%$

- Independencia económica: 33\%

- Estabilidad emocional: $33 \%$

- Sentimiento de culpa: $33 \%$

- Expectativas de futuro: $33 \%$

Analizando las respuestas, no concuerdan con sus demandas. Curiosamente, el tema de la autoestima es muy solicitado y en cambio menos valorado. Interpretamos este resultado como que les cuesta identificar y reconocerse en su autoestima; por poner un ejemplo, una de las mujeres del grupo se sentía muy intimidada cuando las conductoras con su mirada, escucha y su silencio, le daban protagonismo prestando atención a su relato; la mujer no se reconocía. Otra explicación a esta valoración es que se trató el tema más específicamente al principio, en la primera etapa.

Los ejercicios individuales son los que menos valoran; el rol-playing y las escenificaciones están más valoradas, al ser más activos y participativos.

Otro aspecto sorprendente para las profesionales es la no puntuación en los temas que ellas mismas expusieron: la amistad, el ocio, el futuro y el trabajo.

Igual que otros temas, como el de la sexualidad, con un $16 \%$ solamente, cuando tratamos los abusos, y las exposiciones fueron muy íntimas, pero también a partir de este tema una de las integrantes hizo una fuga y dejó el grupo. La salud es otro tema que ni puntúan, cuando el médico venía cada jueves a finalizar el grupo con los ejercicios corporales de Qi-gong, pero al mismo tiempo explicaban lo bien que les iban estos ejercicios y los repetían en sus casas. Un resultado contradictorio, quizá por no saber expresar verbalmente la importancia de la salud, también por la gran dificultad de valorarla para sí misma y cuidarse que tienen muchas mujeres que sufren maltratos, extendiéndose a todas las mujeres en general, por los datos de salud de las mujeres que llegan a Servicios Sociales $(70 \%$ de demandas femeninas con estado de salud muy deteriorado $)^{2}$.

2 SIBSS ( Sistema informática básico de Servicios Sociales. Datos del Ayuntamiento de Barcelona, 2007-2008). 
Las valoraciones cuantitativas a las conductoras son muy altas: respuestas y aclaraciones: $100 \%$ muy bien; las ideas y contenidos: $83 \%$ muy bien; facilitar la participación: $83 \%$ muy bien; moderar los turnos de palabras: $66 \%$ muy bien; el ambiente: el $66 \%$ lo valoran muy cercano, acogedor, colaborador y participativo, y el $50 \%$ flexible.

A nivel cualitativo lo que más les ha gustado de la experiencia grupal se reparte entre la parte social hacia las otras, la gente conocida, las compañeras, su humanidad, sentir otros testimonios-, y la parte más individual, personalizándolo en seguir adelante, valorarse más, sentir que no estás sola.

Además de la manera de decir las cosas por parte de las conductoras, la simpatía del médico que dirigía la parte física y corporal de qi-gong, como si no tuviera nada que ver con la salud tradicional (médico de cabecera que receta).

Respecto a lo que cambiarían, todas coinciden en que se hace corto (es medio año): mejorarían la sesión de presentación del primer día, aunque no especifican el qué, y también querrían otra profesional para hablar de sus hijos.

En cuanto a las propuestas a mejorar, proponen empezar más tarde, retrasando el horario porque tenían que ir con prisas con sus hijos.

Más tiempo de duración y un espacio de guardería más adecuado para niños menores de 3 años.

¿Qué otros temas trabajarían? Todos les parecieron muy adecuados, además propusieron el perdón (les pasamos un articulo respecto a este tema, que fue tratado por encima y en las últimas sesiones).

En cuanto a la valoración cualitativa de su estancia en el grupo, era muy positiva: se sintieron: "Comprendidas y arropadas", "Primero muy mal, luego mejor", "Valorada y estimada por compañeras y monitoras", "Muy cuidada desde el primer día", "Muy bien". Incomodas el 50\%, "al principio porque es muy duro hablar de las experiencias vividas".

Las "cosas" que te ha aportado la experiencia grupal: "Apoyo"." Lo que yo he pasado y estoy frenando, les ha servido a las demás para aclararse de lo que les pasa". Comprensión y acogimiento.

Las "cosas" que has recibido: "Estabilidad y creer más en mî", "más independencia", "poderme des- ahogar y hablar sin miedo", "mucha ayuda para salir adelante y mucha fuerza", "apoyo emocional y sentirme más segura".

¿De qué te ha servido venir y participar en el grupo?: "Conocer a otra gente con mi mismo problema". "Para adquirir más autoestima". "Para sentirme valorada por los demás, aunque por él no".

Como valoran la relación con las compañeras: En general: "buena", "bonita", "querría seguir con la relación”.

¿Qué me ha hecho daño o causado dolor?: "Recordar lo que quiero olvidar". "Al principio recordar dolió mucho". "La ansiedad que me ha causado".

¿Piensas que sería útil o necesaria tu continuidad en el grupo, y por qué? "Si, para continuar dando y recibiendo experiencia". "Para acabar de confiar en mí misma". "Porque me han enseñado a valorarme". "Ha sido corto y nos queda mucho dolor dentro". "Porque tengo miedo de que cuando no venga, vuelva a ser como antes."

En cuanto a las conductoras, la valoración es altamente positiva: "Como pez en el agua de gusto y bien". Las profesionales acogen con mucha satisfacción estos resultados en la evaluación y analizan que siempre necesitan más tiempo, más sesiones, nunca da tiempo a todo, a acabar los temas, pero son 20 sesiones, seis meses, semana a semana, cohesionándose el grupo, viendo el avance, período a período, sorprendiendo de repente en que se acaba el tiempo y se ha de preparar el cierre, lamentando que la intervención llega a su fin, cuando ya todo es más fácil y, superados algunos miedos, todo rueda con más agilidad, con más tranquilidad.

Se experimenta, a nivel perceptivo, un salto grande en todas, también a través de los cuestionarios de autoestima (escala de Rosenberg, 1965), en ella no han mejorado muchos puntos de la primera pasación, puede ser un mecanismo de defensa, autovalorarse de forma alta al inicio (autoestima no real), de auto contención para no hundirse más.

O al menos, no se correspondía a la realidad física y mental en la que se encontraban en aquel momento.

Una vez finalizado el grupo, quedan atrás otros ejercicios, sin tiempo a aplicarlos, pero sí se han dado otros a partir de las aportaciones de las propias 
Tabla 2. Resultados de la escala de autoestima de Rosenberg en tres tiempos

\begin{tabular}{cccc}
\hline Mujeres/tiempos & febrero & Junio & noviembre \\
\hline M & 20 & 19 & 20 \\
I & 13 & 18 & 24 \\
K & 21 & 23 & 25 \\
D & 18 & 24 & 23 \\
G & 22 & 23 & 18 \\
E & 20 & 20 & 18 \\
\hline
\end{tabular}

mujeres que son los que enriquecen la evolución y el crecimiento grupal.

\section{Evaluación}

En el Distrito realizamos una evaluación anual todas las profesionales que intervienen con mujeres, con la técnica de la Mujer, sobre las diferentes intervenciones realizadas. En esta se configura el nuevo equipo que creará y conducirá el próximo grupo.

Al principio, el trabajo en equipo de construcción de un grupo es de un grado muy elevado, parece mucho trabajo, por las derivaciones, reuniones, coordinaciones y entrevistas, pero a medida que avanza la intervención, pasada la primera etapa de contención, una vez configurado y cohesionado el grupo, el trabajo se hace más llevadero, ágil, sistemático y satisfactorio, hasta el cierre se convierte en una parte fácil y constructiva con su parcela divertida.

En este Distrito se valora y se tiene en cuenta la carga de trabajo que significa el grupo, y favorece, facilita y prioriza en las profesionales del equipo el trabajo grupal a realizar, expectante a la vez de los resultados. El equipo que ha llevado el grupo retorna la información al resto de profesionales y técnicas del Distrito, donde se exponen las derivaciones, analizando tanto la cantidad como el contenido e idoneidad para la integración al grupo, y los servicios de red que han derivado, evaluando su comprensión y necesidad de la intervención en grupo por el resto de servicios de las zonas y los resultados evaluados por las profesionales y los respondidos por las mujeres en los cuestionarios.

A nivel metodológico, el cierre también es una parte muy valorada, tanto por el equipo como por las mujeres.
- Las mujeres al finalizar la intervención continúan vinculadas a sus profesionales referentes (derivantes al grupo)

A los 5/6 meses se las vuelve a citar para una evaluación post-grupal e intermedia, evidenciándose que en el $80 \%$ la evolución es positiva.

\section{Profesional experta y externa al Servicio}

Desde el Sector (Área de planificación central) centralizan las intervenciones grupales de las diferentes zonas y distritos, ofreciendo soporte a los grupos con una supervisión externa, especializada en dinámica y en conducción grupal.

Se realizaron dos sesiones de supervisión de una hora y media. En el primer tiempo, en marzo, se habían realizado seis sesiones del grupo. La segunda sesión de supervisión fue en junio, justo dos semanas antes de finalizar el grupo.

En la primera se supervisaron las derivaciones y los criterios de selección. Preocupaban varios aspectos: las derivaciones, algunas no muy acertadas, la estructura de las sesiones y la ansiedad del grupo.

La supervisora aportó un registro de evaluación grupal en tres tiempos, inicial, intermedio y final para evaluar la evolución de la dependencia-independencia de las mujeres.

En la segunda sesión de supervisión, finalizando el grupo, se trataron las fugas de último momento (concretamente de dos mujeres que no llegaron al final), y el cierre con la nueva metodología de incorporación de las mujeres en la conducción grupal.

\section{Conclusiones y propuestas de futuro}

Las atenciones en violencia de género en Ser- 
vicios Sociales son cada vez más frecuentes, la demanda femenina es alta y los-as profesionales cada vez estamos más atentas y nos formamos en este tema preocupante y a la vez de freno para el avance de la igualdad entre hombres y mujeres.

A parte de la intervención individual, la grupal acelera el proceso de curación y recuperación para salir del maltrato y avanzar en la decisión de vida de muchas mujeres, pasando de posición de victimas a supervivientes reforzadas y con ganas de salir adelante.

Es importante amplificar esta metodología de intervención, a la vez que enriquecer el trabajo de construcción de equipos profesionales y fortalecer el viejo esquema de prevención comunitaria que debería resurgir más en el futuro de los Servicios Sociales.

Es como cerrar el círculo, el grupo hace de puente entre la mujer y su resurgir, fortalecida hacia la comunidad, cada vez mejor preparada para integrar a la mujer en la dinámica de avance sobre las desigualdades.

La valoración de los resultados, partiendo no solo de la escala de autoestima, que en casi todas asciende, es que en general la situación de las mujeres mejora, se cuidan más, están más fuertes, pasan a la acción, buscan trabajo, algunas se separan, ponen límites al maltrato, revisan sus relaciones tanto con su familia como con sus hijos, si los tienen, como también, si se da el caso, se replantean nuevas relaciones futuras y su posición es más fuerte.

De ahí la importancia de la intervención grupal en situaciones de maltrato, que se convierte en prevención e inversión para su futuro en el mundo femenino y en la sociedad en general, mejorando la salud, el trabajo y las relaciones. Otro aspecto importante es que sepan pedir ayuda y mantener si hace falta por algún tiempo más su relación con las profesionales referentes, porque sabemos por experiencia que algunas, al cabo de un cierto tiempo, toman decisiones importantes.

Por tanto, el "tempus" del grupo sigue funcionando y se perpetua dando resultados a largo plazo. Esta experiencia es clave, ya que crea en el mundo de muchas de las mujeres integrantes un antes y un después de la asistencia al grupo.

\section{Extended Summary}

This group intervention is presented as a practice experience that works with women who are abuse by their partners. It works with their improvements, their evolution and also the importance of being part of a Global Project that involves both women and professionals who form a networking, of a community outreach and city.

The project focuses on a specific territory, a district called Horta-Guinardó, one of the ten that form the city of Barcelona. It is divided into four zones; each has got a center of social services. It consists of professionals of different disciplines as social workers, social educators and psychologists.

The configuration of the team is found within the general goals and it is the first one. It is formed by various professionals of the four areas. These are formed specifically on the issue of violence gender. They get involved from the detection and the personal referral to the methodology group. Highlighting the importance at this intervention of interdisciplinary, the team is formed by a psycholo- gist who is expert on emotional issues arising from situations of gender abuse and also dynamics groups. Two social workers as experts in resource management and in group dynamic of women in this situation. One as a co-host and the other one as an observer to ensure training and participation.

Second goal is the participation of all women from all over the district. Although the group is made in a specific area - Guinardó- to strengthen and improve networking as a third objective, reaching the maximum of women as possible and strengthen the unification of criteria to better referral between different professionals and services.

The fourth goal focuses on enhancing the core value of self-esteem, so cross, throughout the group intervention for women members, while to reduced the abuse they suffer at the hands of their partners.

They will improve their situation at different levels, as we will discuss in the final results. This is the most important objective to get inside the 
group intervention. Finally the fifth goal develops the independence and autonomy of women, introducing methodology and innovation in the group closure. It consists on the incorporation of women in the conduct of last sessions, speeding the grieving process and showing the empowerment gained by them.

Methodology; as we initiated , as configured, and advocate the participation, as we build the sessions , as we value, etc. It acquires a fundamental part in this article. It wants to show not only the temporal process behaves, interdisciplinary teamwork, professional growth and networking outreach to women, improving their situation and condition , but also the methodological developments, steps to follow and improve for ten years experience.

We begin with the circuit of derivations, reviewing the used material and if it's possible to improve the referral form, especially to be better understood for the different referring professionals from other services or other zones.

The registry of interview to value the suitability of women derived to the group, as the same time as to extend the information, to dilute its resistance to them and to motivate them for its participation. Sometimes these interviews must be done by two of the professionals who formed the team, according to the gravity of the situation and the state of the derived women.

Throughout years of experience, we've been able to state that we explored the state of health of many of the women; a high percentage suffers all type of muscular ailments, pains, menstrual upheavals, sometimes tumors of female physiology, backaches... Together with the high emotional load of sessions, at preventive level, it is considered to close them with corporal space, qi-gong exercises, respiratory and movement exercises which help to mobilize blood supply. The exposition and the story telling of such a high emotional level could cause a contraction of the blood vessels. These exercises are always guided by a specialist of Chinese classic medicine.

In the configuration and the construction of this group project is important to know that many of these women are mothers. They have got young children and they are highly responsible about them, at a high level both of conflict and alliance. So at the same time, a parallel children's area is created during the same hours.

In another nearby space in the center, there's another professional team. It is formed by two educators or monitors. They are in charge of defining the children's area in a very coordinated way. It is not a simple space; the most important objectives are the observation, containment and assessment regarding the impact towards these children of the situation experienced by their mothers.

The intervention present profiles of different women who take one of the part of the group experience, 9 women in total. The variables above are; zone and serviced area , age and country of origin, if they've got children, employment status, their health and at last the types of abuse every one of them have suffered.

We analyze the phases in 3 blocks. There are 20 sessions from February to June. The first stage covers from the first session until the ninth, where the incorporation of new women is closed. At this time we analyze the creation of the group, the group cohesion, the initial stories, the pain and the suffering shared, containment. It's the most difficult stage; it is so for the initial content stories, for the anxiety of the group and also for the created expectations and the professional approach.

The second stage covers from the tenth session to the sixteenth. It coincides with the return of the Easter holidays. It takes some time to be on its own and also for reflection. Women are beginning to be more aware of the group effects. They want to reconnect. It is the most productive stage. This stage repairs and they have more contact with themselves and they start to leave behind their victimization. The group acquires body and identity.

Finally the third stage, the last four season of closure and at the same time of overture. It starts the active women's participation in the conduction and closure of the group. This is the autonomy stage, greater security and independence, and it also is the last exercise in empowerment.

The evaluation and the results were made both ways; quantitatively and qualitatively. Quantitatively made with a questionnaire and qualitatively made through an oral presentation at the last session. 
$86 \%$ of all women answered the questions. Now we want to show the most significant quantitative answers.

The overall assessment, the intervention was considered more appropriate by $66 \%$ and the remaining $33 \%$ quite adequate.

The exercises and works within the group were also assessed as more adequate and also the personal stories were important for the group.

Highlight the assessment made by external professionals who visited us. They assessed our work as very suitable by $83 \%$.

It was only $50 \%$ of valuation to its control, the other $50 \%$ was considered less appropriate.

The space, the environment, time were also assessed as very adequate by $66 \%$, and the numbers of attendees assessed as very adequate by $83 \%$.

The higher qualifications were directed to the professionals, with our answers and clarifications. $100 \%$ felt that we did it very well, as well the ideas and the content, and in facilitating the participation by $83 \%$.

Only by observing the quantitative results, we can analyze the high difficulty of these women who have suffered abused, when they have to valuate themselves. The way they valuated us so high it could be a way to thank us, the professionals. But the chart reviews of self-esteem of Rosenberg's scale; it also indicates the difficulty increase in the final results.

If although they show significant improvement in their qualitative assessment, they have felt understanding, comprehension, appreciation, esteem, healing, being able to talk about their experiences, and sharing all that with their classmates (being this the most valued social part).

They feel that the group has given them support. And this helps them to have things more clear in mind. They have received comprehension, understanding, stability, believe in themselves, independence. It has helped them to meet other people who have the same problems, to acquire more selfesteem and to feel more valuated by others.

In the post group intermediate assessment, after five or six months, women have changed in different aspects, employment, training, housing, some of them have removed from their partners. It is reveal- ing a positive development by $80 \%$ of them. Most of them leave our services and stop having contact with us.

As a conclusion and future proposals, being more often the attention to women who suffered genre violence in the social services, the group accelerates the healing process and their recovering to be outside the circle of abuse of their partner or ex partner. They want to keep on moving to their goals and they take decisions. They change their victim position to enhanced survivor.

We propose to amplify the intervention methodology, because while enhancing the construction work of the professional team, it strengthens the network of services and promotes community prevention.

The group is used as a bridge between women and their strengthened resurgence to the community.

In general women situation improves. They take more care of themselves, they are stronger, they take action, they seek for work, and they sit limits to the abuse. Some of them split with their partners. They review their relation both with the family and with their children. They also rethink about new future relations where their position is stronger.

The group intervention with women who suffer abuse becomes a future investment in the society and in the world. The search of equality and respect is part of their values, improving their life quality and their health.

The tempus group keeps on working. We know that a certain time women make important decisions, giving these decisions long term results. This experience is very important, it establishes a before and an after to these women who have taken part of the group.

\section{Referencias}

Antons, K. (1990). Practica de la dinámica de grupos. Barcelona: Herder.

Ansíen, D. (2004). El grupo y el inconsciente. Madrid: Biblioteca Nueva.

Bach, E. y Darder, P. (2002). Sedúcete para seducir. Barcelona: Paidós.

Bion, W. R. (1980). Experiencias en grupo. Barcelona: Paidós. 
Cembranos, F. y Medina, J. A. (2003). Grupos inteligentes. Madrid: Popular

Corsi, J. (2002). Violencia Masculina en la pareja. B. Aires: Paidos

Echeburua, E. y Corral, P. (2006). Manual de violencia familiar. Madrid: Ed. Siglo XXI.

Fritzen, S. J. (1988). 70 Ejercicios prácticos de dinámica de grupos. Santander: Salterrae

Linares, J. L. y Campo, C. (2000). Tras la honorable fachada. Barcelona: Paidós.

Lorente, M. (2001). Mi marido me pega lo normal. Barcelona: Ares y mares. Ed.Crítica.

Lorente, M. (1999). Agresión a la mujer. Granada: Comares.

Lorente, M. (2004). El rompecabezas. Barcelona: Ares y mares. Ed. Crítica.
Masip J. Martinez.Ch. (2002). Cenicienta tiene un mal sueño. Barcelona: Ayuntamiento de Barcelona.

Masip, J. y Martinez, Ch. (2002). Violencia domestica. Cuadernos para profesionales. Barcelona: Ayuntamiento de Barcelona.

Matthew, M. y Fanning, P.(2002). Autoestima. Evalua-ción y mejora. Barcelona: Martinez Roca.

Pichon-Rivière, E. (1985). El proceso grupal. B. Aires: Nueva visión

Roca, N. y Masip, J. (2010). Intervención grupal. Violéncia sexista. En prensa. Barcelona: Herder.

Shinoda Bolen, J. (2004). El millonésimo circulo. Barcelona: Kairós.

Vanistendael, S. y Lecome, J. (2002). La felicidad es posible. Barcelona: Gedisa.

Manuscrito recibido: $11 / 02 / 2010$

Revisión recibida: 15/02/2010

Manuscrito aceptado: 20/02/2010 